\title{
Distributed Inference Networks with Costly Wires
}

\author{
Lav R. Varshney \\ Laboratory for Information and Decisions Systems \& Research Laboratory of Electronics \\ Massachusetts Institute of Technology
}

\begin{abstract}
Distributed control systems are physical constructs, incurring deployment and maintenance costs for their communication infrastructure. Inference is a central function of many distributed control systems. This paper formulates and studies the tradeoff between algebraic notions of inference functionality and algebraic notions of wiring costs. It is shown that separated topology design and node placement yields optimal network design. This design problem is shown to be NP-complete, but is carried out for small network size. A natural relaxation is shown to be a reverse convex minimization problem.
\end{abstract}

\section{INTRODUCTION}

Control, communication, and computation networks take physical form and are constrained by all that is physically manifest. There are costs in building, maintaining, and operating networks, such as the cost of deploying and maintaining the physical infrastructure of network links: wiring costs. Wiring costs may limit system performance but most previous works have ignored the fact that networks must be laid out in space, cf. [1], [2]. This paper formulates novel tradeoffs between algebraic notions of synchronizability [3], [4] and wiring cost for distributed inference networks.

A primary aspect of the problem formulation is drawing a graph in physical Euclidean space, a basic problem in computer science [5]. Although there are several theories of graph drawing in spaces, an adjacency model where the geometry respects the binary relations of adjacency/nonadjacency between vertices is used here. In particular, this paper considers graph drawing in Euclidean space to minimize link length [6], [7], a method which also has certain aesthetic appeal [8], [9].

Two network design problems are formulated using two related notions of synchronizability [10]. For the first, functionality is measured by the algebraic connectivity of the network, which determines the convergence speed of several distributed consensus algorithms on graphs [3]. For the second, functionality is measured by the eigenratio of the network, which determines the convergence speed of several other distributed inference algorithms on graphs [4], [11], [12]. For both formulations, there is separation such that optimal networks are found by solving two subproblems: designing the graph connectivity and then placing the graph in physical space. It is further shown that the graph design problem is NP-complete and that the natural relaxation is a reverse convex program.

Supported in part by a National Science Foundation Graduate Research Fellowship, Grants 0729069, 0836720, and 0801549, and a Siemens Corporate Research Grant, "Advanced Control Methods for Complex Networked Systems."
Results obtained from this work may not only have conceptual implications for synthesis of engineered inference networks, but also for analysis of natural systems [7], [13] [15].

The remainder of the paper is organized as follows. Section II reviews certain relevant aspects of algebraic graph theory. Section III comments on the functionality of networked systems and Section IV explicates the wiring cost of a network under optimal Euclidean placement. Section V defines the functionality-cost tradeoffs for networks. Section VI gives a new NP-completeness result; Section VII gives properties of the network design problem and provides small optimal networks; and Section VIII provides a natural reverse convex relaxation that may be useful for designing large networks. Section IX concludes the paper.

\section{ALGEBRAIC GRAPH THEORY}

The eigenvalues and eigenvectors of certain matrices associated with graphs are often of central importance in understanding the properties of systems defined on those graphs.

Let $G=(V, E)$ be a graph with vertex set $V$ of cardinality $n$ and edge set $E \subseteq V \times V$. Let $A$ be the adjacency matrix of the graph. Let $d_{j}$ indicate the degree of vertex $j$ and let $D$ be the degree matrix of the graph, which takes value $d_{j}$ along the diagonal and value 0 otherwise. The Laplacian matrix of a graph, $L$, satisfies $L=D-A$. The eigenvalues of $L$ are denoted $\lambda_{1} \leq \lambda_{2} \leq \cdots \leq \lambda_{n}$. Since $L$ is symmetric, all of its eigenvalues are real and eigenvectors corresponding to different eigenvalues are orthogonal.

The Laplacian matrix of every graph is positive semidefinite and its eigenvalues are non-negative. Furthermore, since the row sums of $L$ are all zero, the all-ones vector $\overrightarrow{1}$ is an eigenvector with eigenvalue $\lambda_{1}=0$. The multiplicity of the eigenvalue 0 determines the number of connected components in the graph; if $\lambda_{2}>0$, then the graph is a single connected component.

The celebrated Courant-Fischer min-max theorems endow the eigenvalues with certain optimality properties. In particular,

$$
\lambda_{2}=\min _{\left\{\vec{x} \in \mathbb{R}^{n}: \vec{x} \neq \overrightarrow{0} \text { and } \vec{x} \perp \overrightarrow{1}\right\}} \frac{\langle\vec{x}, L \vec{x}\rangle}{\langle\vec{x}, \vec{x}\rangle},
$$

where $\overrightarrow{0}$ is the all-zero vector. This second smallest eigenvalue $\lambda_{2}$ is called the algebraic connectivity of the graph. The largest eigenvalue satisfies

$$
\lambda_{n}=\max _{\left\{\vec{x} \in \mathbb{R}^{n}: \vec{x} \neq \overrightarrow{0} \text { and } \vec{x} \perp \overrightarrow{1}\right\}} \frac{\langle\vec{x}, L \vec{x}\rangle}{\langle\vec{x}, \vec{x}\rangle} .
$$


The largest eigenvalue $\lambda_{n}$ is called the spectral radius of the graph Laplacian. The eigenratio is defined as $\rho=\lambda_{2} / \lambda_{n}$.

Several non-isomorphic graphs may share the same Laplacian eigenspectrum, however characterizing such cospectral graphs is difficult [16], [17]. Moreover, there is no precise characterization of how the Laplacian spectrum of a graph changes when edges are added or deleted. There are bounds on changes to the Laplacian spectrum [18]-[20], including ones on the relationship between $\lambda_{2}$ and $\lambda_{n}$ [21].

\section{FunCtionALITY}

The convergence times of inference algorithms and other dynamical processes defined on connected networks are of central interest in distributed control. This section briefly reviews the role that algebraic connectivity and eigenratio play in convergence time. Since only logical aspects of networks (and not physical ones) are dealt with in this section, the Laplacian matrix is a full description.

This work focuses on distributed inference in which all agents receive and agree upon the result of the final computation [22]-[24], rather than the setting in which agents transmit information to a fusion center for final processing [25]-[28].

Over the past few years there has been a growing literature on synchronization of distributed systems and consensus algorithms. Many of these works find that the algebraic connectivity of the network graph determines the speed of convergence and so large algebraic connectivity is desirable, see e.g. [3], [29] and references therein. In an alternate line of work, it has been found that the convergence speed of a linear distributed averaging algorithm is determined by the eigenratio [4], [30]. The convergence speed of a distributed detection algorithm built on this distributed averaging algorithm is also determined by the eigenratio [4]. The eigenratio also plays a role in synchronization of a system of coupled oscillators [11].

Kar et al. assert that the eigenratio is more sensitive to variations in the algebraic connectivity than variations in the spectral radius. They go on to develop topology constructions that optimize $\lambda_{2}$ [4]. Rad et al. demonstrate that graphs optimized for $\rho$ also have large $\lambda_{2}$ [10]. The sequel makes some comparison as to whether networks with large $\lambda_{2}$ also have large $\rho$, once wiring cost constraints are imposed.

A few previous works have considered optimizing $\lambda_{2}$ or $\rho$ through the choice of graph topology [4], [31]-[34]. Ramanujan graphs, a particular class of expander graphs, have been found to have extremal properties [4].

\section{WIRING COSTS}

A physical network in Euclidean space is determined not only by the adjacency matrix but also by the physical placement of the nodes (and thereby the physical lengths and locations of the links). Suppose that edges are created by an infrastructure of wires that have cost that is quadratic in length. The problem of optimal graph drawing under this quadratic infrastructure cost reduces to a problem in algebraic graph theory, as shown in [6], [7].
Consider the wiring cost for a graph with adjacency matrix $A$ and vertices placed on the real line at $\vec{x}=$ $\left(x_{1}, x_{2}, \ldots, x_{n}\right), x_{i} \in \mathbb{R}$. The total wiring cost, $C_{1}$ is given by

$$
\begin{aligned}
C_{1} & =\frac{1}{2} \sum_{i=1}^{n} \sum_{j=1}^{n}\left(x_{i}-x_{j}\right)^{2} a_{i j} \\
& =\frac{1}{2} \sum_{i=1}^{n} \sum_{j=1}^{n}\left(x_{i}^{2}-2 x_{i} x_{j}+x_{j}^{2}\right) a_{i j} \\
& =\sum_{i=1}^{n} x_{i}^{2} \sum_{j=1}^{n} a_{i j}-\sum_{j=1}^{n} \sum_{i: i \neq j} x_{i} x_{j} a_{i j} \\
& =\vec{x}^{T}(D-A) \vec{x}=\vec{x}^{T} L \vec{x} .
\end{aligned}
$$

Two non-triviality constraints should be imposed on the wiring cost minimizing placement. First, all vertices should not be right on top of each other. Second, a normalization that $\vec{x}^{T} \vec{x}=1$ is imposed, so that vertices are not arbitrarily close to being right on top of each other. Under these non-triviality constraints, the Courant-Fischer theorem implies that the placement $\vec{x}$ should be the unit eigenvector associated with $\lambda_{2}$ and that the incurred wiring cost is $\lambda_{2}$, for placement in one-dimensional Euclidean space.

If the network is to be drawn in two-dimensional Euclidean space and the horizontal placement is $\vec{x}$ and the vertical placement is $\vec{y}$, then the wiring cost separates as $C_{2}=\vec{x}^{T} L \vec{x}+\vec{y}^{T} L \vec{y}$. If a further non-triviality constraint that the horizontal placement must be orthogonal to the vertical placement is imposed, ${ }^{1}$ then the optimal placement has $\vec{x}$ be the eigenvector associated with $\lambda_{2}$ and $\vec{y}$ be the eigenvector associated with $\lambda_{3}$. The incurred cost is $\lambda_{2}+\lambda_{3}$. In three dimensions, the cost is $C_{3}=\vec{x}^{T} L \vec{x}+\vec{y}^{T} L \vec{y}+\vec{z}^{T} L \vec{z}$ and under optimal non-trivial placement, the cost is $C_{3}=$ $\lambda_{2}+\lambda_{3}+\lambda_{4}$.

One can note the following separation principle [6], [8]:

Proposition 1: There is an optimal non-trivial placement of graph vertices in Euclidean space for any given choice of graph topology $A$.

Proof: The result follows directly, since for each $A$ there is an optimal eigenplacement that incurs cost

$$
C_{r}(A)=\sum_{i=2}^{r+1} \lambda_{i}(A)
$$

for embedding in Euclidean space $\mathbb{R}^{r}$ for any $r=1, \ldots, n-$ 1.

In practice a network will be embedded in $\mathbb{R}^{2}$ or $\mathbb{R}^{3}$. Of course smaller cost is desirable.

\section{Functionality-Cost TradeofF}

Having defined network functionality in terms of either $\lambda_{2}$ or $\rho$ and having defined network cost in terms of optimized wiring costs in Euclidean space $C_{r}$, the interesting tradeoff

\footnotetext{
${ }^{1}$ If the network is to be performing detection, estimation, or control on a bandlimited field, a reason for imposing this non-triviality constraint may be related to sampling. Stable sampling requires the entire space to have reasonable sampling density [35].
} 
between the functionality and cost is studied. Large $\lambda_{2}$ is desirable to enhance functionality whereas small $\lambda_{2}$ is desirable to reduce costs. If the optimization problem were written in Lagrangian form, one could say that the cost term promotes networks where spatially close vertices are connected, whereas the functionality term promotes long range links; in some sense, the cost-functionality tradeoff involves trading off of the number of long range links.

Following Proposition 1, the network design problems optimize graph topology and involve spectral graph theory. Fix the number of vertices of the graph $\Gamma$ at $n$ and denote the Laplacian as $L(\Gamma)$. Also fix an $r, 1 \leq r<n$, as the dimension of the Euclidean space, e.g. $r=2$, or $r=3$.

Problem 1 (Algebraic Connectivity): Find the following functionality-cost function:

$$
s_{\lambda}\left(C_{r}\right)=\max _{G \in \mathcal{G}\left(C_{r}\right)} \lambda_{2}(L(G))
$$

where

$$
\mathcal{G}\left(C_{r}\right)=\left\{\Gamma: \sum_{i=2}^{r+1} \lambda_{i}(L(\Gamma)) \leq C_{r}\right\} .
$$

Also find optimizing graphs

$$
G_{\lambda}^{*}\left(C_{r}\right)=\underset{G \in \mathcal{G}\left(C_{r}\right)}{\arg \max } \lambda_{2}(L(G)) .
$$

When $r=1$, the objective function and the constraint coincide. Primary interest here, however, is in $r=2$ or $r=3$.

Problem 2 (Eigenratio): Find the following functionalitycost function:

$$
s_{\rho}\left(C_{r}\right)=\max _{G \in \mathcal{G}\left(C_{r}\right)} \rho(L(G))=\max _{G \in \mathcal{G}\left(C_{r}\right)} \frac{\lambda_{2}(L(G))}{\lambda_{n}(L(G))},
$$

where $\mathcal{G}\left(C_{r}\right)$ is as in (2). Also find optimizing graphs

$$
G_{\rho}^{*}\left(C_{r}\right)=\underset{G \in \mathcal{G}\left(C_{r}\right)}{\arg \max } \rho(L(G))=\underset{G \in \mathcal{G}\left(C_{r}\right)}{\arg \max } \frac{\lambda_{2}(L(G))}{\lambda_{n}(L(G))} .
$$

One might also consider the opposite problems of finding $C_{r}\left(s_{\lambda}\right)$ and $C_{r}\left(s_{\rho}\right)$. There may be slight differences, just like there are slight differences between the cost-distortion function and the distortion-cost function in information theory [36].

Problems 1 and 2 formalize optimization of inference functionality under infrastructure cost constraints. There has been some previous work looking at tradeoffs between functionality and cost in physical networks. For spatial distribution networks, the relationship between the lengths of paths from each vertex to the root vertex and the sum of the lengths of all edges in the network is discussed in [2]. It has been suggested that neuronal networks are not exclusively optimized for minimal global wiring, but also for factors including the minimization of processing steps [15]. ${ }^{2}$ The tradeoff between algorithm performance and communication cost has also been discussed as a network design problem [37]. Ghosh and Boyd briefly discuss optimizing $\lambda_{2}$ when costly links may be added to a network [31].

\footnotetext{
${ }^{2}$ Note however that when talking about 'processing,' average path lengths rather than some measure that takes actual computations into account are used; see also [13], [14].
}

\section{OPTIMIZATION IS HARD}

The problems as defined are optimizations over graph Laplacians, which are discrete objects. A Laplacian is symmetric, positive semidefinite, each row sums to zero, and its off-diagonal elements are either zero or minus one. Conversely, if $L$ is any $n \times n$ matrix that satisfies these conditions, then it is the Laplacian of some graph on $n$ nodes [38]. The set of Laplacian matrices

$$
\left\{L \in \mathbb{R}^{n \times n}: L=L^{T}, L \succeq 0, L \overrightarrow{1}=0, L_{i j} \in\{0,-1\}, i \neq j\right\}
$$

is denoted by $\mathcal{L}$. Since $\mathcal{L}$ is a discrete space, the optimization problems are integer programs, which are often difficult to solve.

The algebraic connectivity with wiring costs problem may be studied using computational complexity theory [39]. Consider the decision version of the optimal algebraic connectivity optimization problem without any wiring cost constraints.

Problem 3: MAXIMUM Algebraic Connectivity Augmentation

- Given an undirected graph $G=(V, E)$, a non-negative integer $k$, and a non-negative threshold $t$,

- Seek a subset $A \subseteq E^{c}$ of size $|A| \leq k$ such that the graph $H=(V, E \cup A)$ satisfies $\lambda_{2}(H) \geq t$.

Theorem 1 ( [40]): Problem 3 is NP-complete.

Now impose wiring costs.

Problem 4: MAXimum Algebraic Connectivity Augmentation With Wiring Costs

- Given an undirected graph $G=(V, E)$, a non-negative integer $k$, a non-negative threshold $t$, and a non-negative wiring cost $C_{r}$,

- Seek a subset $A \subseteq E^{c}$ of size $|A| \leq k$ such that the graph $H=(V, E \cup A)$ satisfies $\lambda_{2}(H) \geq t$ and $\sum_{i=2}^{r+1} \lambda_{i}(H) \leq C_{r}$.

Theorem 2: Problem 4 is in class NP.

Proof: Eigenvalues of a matrix can be computed in polynomial time, e.g. using Gaussian elimination which is $O\left(n^{3}\right)$.

Lemma 1: The complete graph on $n$ vertices, $n>2$ has the following Laplacian eigenspectrum: $\lambda_{1}=0, \lambda_{2}=\cdots=$ $\lambda_{n}=n$.

Now to give the main result of this section.

Theorem 3: Problem 4 is NP-complete.

Proof: Problem 4 is in class NP, by Theorem 2. Moreover, one can restrict Problem 4 to Problem 3 by only allowing instances having $W_{d}=n d$. This is the largest wiring cost possible for a graph with $n$ vertices (achieved by a complete graph, Lemma 1). Since Problem 3 is a special case of Problem 4 and it is NP-complete (Theorem 1), the result follows [39].

\section{Some Solutions}

In studying optimal network design, one may list all connected unlabeled graphs on $n$ nodes [41, A001349] and calculate their cost and functionality values [42]. Since cospectral graphs exist, the number of graphs that need to be checked is smaller than the number of connected 


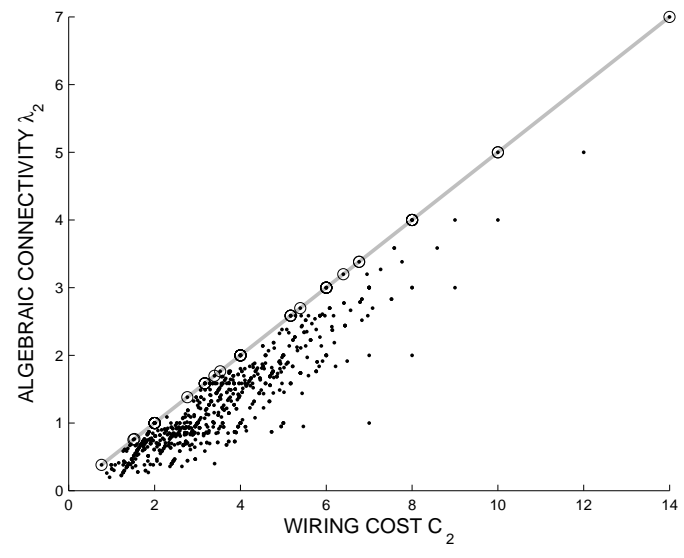

Fig. 1. Algebraic connectivity as a function of quadratic wiring cost under optimal non-trivial placement in $\mathbb{R}^{2}$ for all connected graphs on $n=7$ vertices. The upper convex hull of these achievable functionality-cost points is denoted by the gray line. Points on the upper convex hull are circled.

unlabeled graphs; the number of unlabeled graphs with distinct Laplacian spectra are given in [17, Table 1]. Rather than using the sense of optimality that had been defined above, consider a more restrictive sense of optimality where points on the upper convex hull of functionality-cost pairs are sought.

Results for $n=7$ are computed and shown in the figures. Figure 1 shows $\lambda_{2}$ as a function of 2-dimensional wiring cost $C_{2}$; the upper convex hull is the straight line $r \lambda_{2}=$ $C_{r}\left(2 \lambda_{2}=C_{2}\right)$. Figure 2 shows $\lambda_{2}$ as a function of 3dimensional wiring cost $C_{3}$. Again, the achievable straight line upper bound $r \lambda_{2}=C_{r}\left(3 \lambda_{2}=C_{3}\right)$ is evident. In fact, this upper bound always holds and is always achievable.

Proposition 2: Solutions to Problem 1 satisfy

$$
s_{\lambda}\left(C_{r}\right) \leq \frac{C_{r}}{r}
$$

and there exists at least one network that achieves the bound with equality for any admissible $n$ and $r$.

Proof: Choose an admissible pair $n$ and $r$. The bound

$$
r \lambda_{2} \leq \sum_{i=2}^{r+1} \lambda_{i}
$$

follows directly from the ordering $\lambda_{2} \leq \lambda_{3} \leq \cdots \leq \lambda_{n}$, and therefore optimal graphs must obey

$$
s_{\lambda}\left(C_{r}\right) \leq \frac{C_{r}}{r}=\frac{1}{r} \sum_{i=2}^{r+1} \lambda_{i} .
$$

The bound is achievable with equality for complete graphs, which have $r \lambda_{2}=C_{r}=n r$, as follows from Lemma 1 .

Figure 3 shows the eigenratio as a function of $C_{2}$ and Figure 4 shows the eigenratio as a function of $C_{3}$.

The five optimal $\mathbb{R}^{2}$ networks in the sense of upper convex hull for the eigenratio are shown in Figure 5; these are drawn in a minimum wiring cost configuration. It turns out that the optimal networks for eigenratio are a strict subset of the optimal networks for the corresponding algebraic

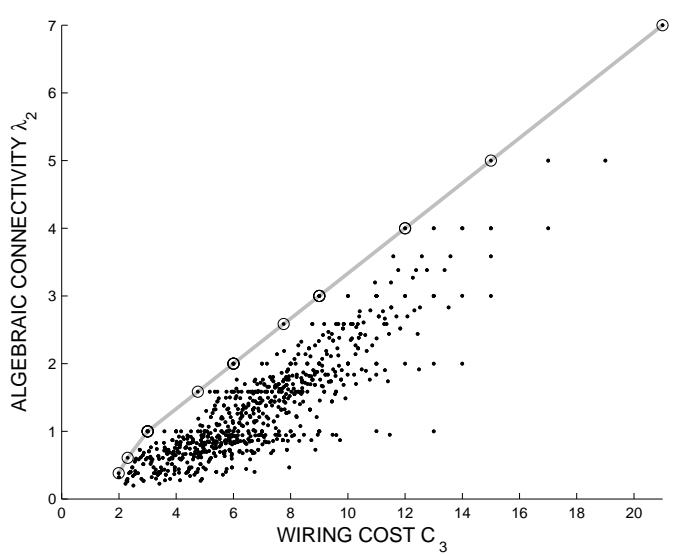

Fig. 2. Algebraic connectivity as a function of quadratic wiring cost under optimal non-trivial placement in $\mathbb{R}^{3}$ for all connected graphs on $n=7$ vertices. The upper convex hull of these achievable functionality-cost points is denoted by the gray line. Points on the upper convex hull are circled.

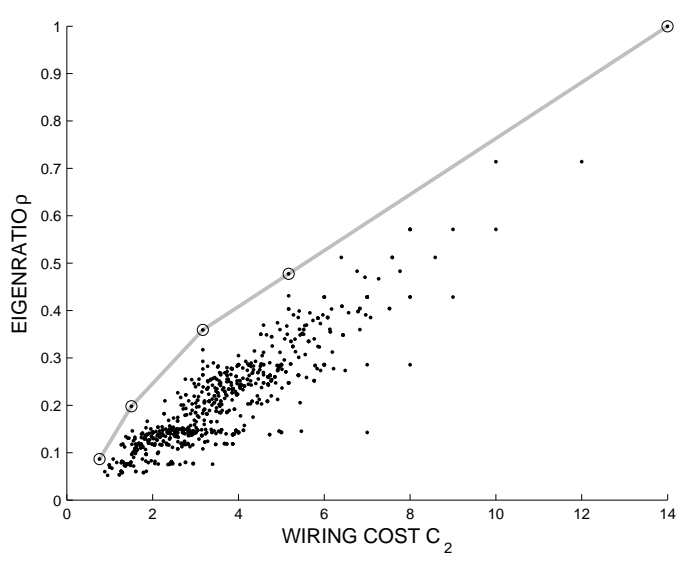

Fig. 3. Eigenratio as a function of quadratic wiring cost under optimal non-trivial placement in $\mathbb{R}^{2}$ for all connected graphs on $n=7$ vertices. The upper convex hull of these achievable functionality-cost points is denoted by the gray line. Points on the upper convex hull are circled.

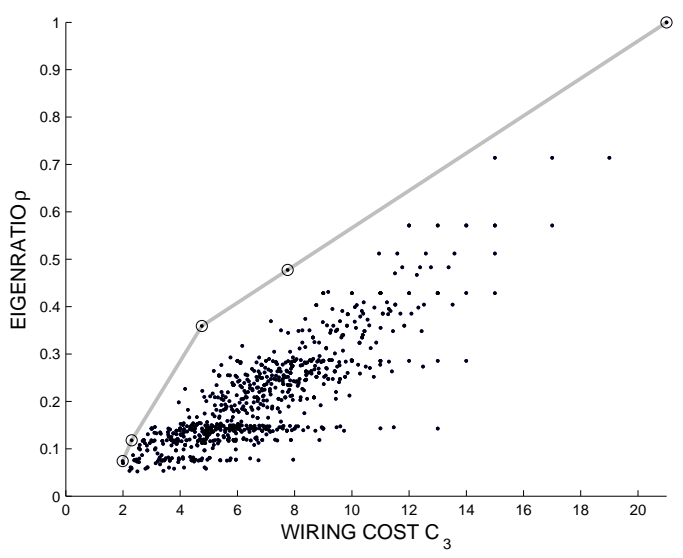

Fig. 4. Eigenratio as a function of quadratic wiring cost under optimal non-trivial placement in $\mathbb{R}^{3}$ for all connected graphs on $n=7$ vertices. The upper convex hull of these achievable functionality-cost points is denoted by the gray line. Points on the upper convex hull are circled. 


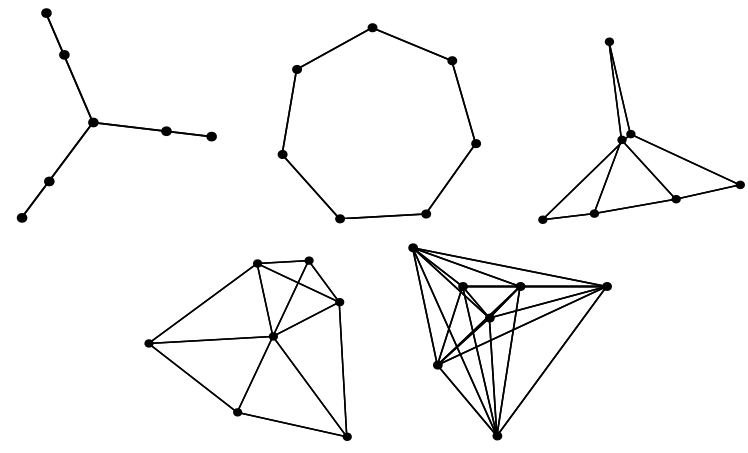

Fig. 5. All networks that are on the upper convex hull of achievable eigenratio-cost points in $\mathbb{R}^{2}$. Networks drawn in a minimal wiring configuration (note that this may not be unique, even up to translation/rotation if some eigenvalues have multiplicity greater than 1 ).
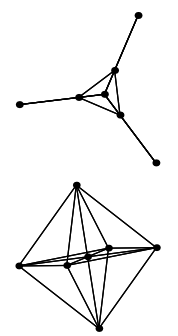
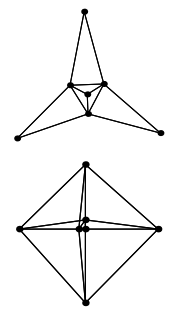
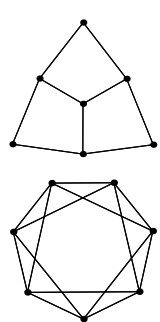
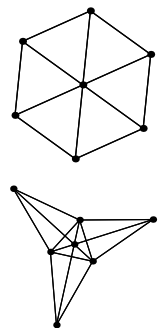

Fig. 6. Some other networks that are on the upper convex hull of achievable algebraic connectivity-cost points in $\mathbb{R}^{2}$. Networks drawn in a minimal wiring configuration (note that this may not be unique, even up to translation/rotation if some eigenvalues have multiplicity greater than 1).

connectivity problem. There are 62 non-isomorphic networks that lie on the upper convex hull; some of these networks are shown in Figures 5 and 6.

The fact that the optimal networks for Problem 2 are a strict subset of the optimal networks for Problem 1 with $n=$ 7 reconfirms assertions that optimizing $\lambda_{2}$ leads to optimal $\rho$ [4], [10]. This subset property does not hold for larger $n$, but is still approximately true [43].

\section{NATURAL RELAXATION}

Although the previous section presented some general results, the focus was on exact solutions for small networks. Exhaustive numerical solutions may be determined for larger numbers of vertices, but the task of listing graphs on $n>10$ vertices becomes computationally difficult.

Given that even neuronal networks of small organisms [13], [44], simple networks-on-chip [45], and small wired sensor networks have $n \gg 10$, there is value in studying the natural relaxation to Problem 1, which may be useful for large networks.

Let $\mathcal{C} \mathcal{L}$ be the convex hull of the set of all Laplacian matrices, $\mathcal{L}$. $\mathcal{C} \mathcal{L}$ is the set of symmetric, positive semidefinite matrices, with zero row sums, and off-diagonal elements between minus one and zero:

$\left\{L \in \mathbb{R}^{n \times n}: L=L^{T}, L \succeq 0, L \overrightarrow{1}=0,-1 \leq L_{i j} \leq 0\right.$ for $\left.i \neq j\right\}$

Essentially invoking the variational form of the CourantFischer idea, one can show that $\lambda_{2}$ is a concave function.
Proposition 3 ( [38]): The algebraic connectivity $\lambda_{2}$ is a concave function of $L$ on $\mathcal{C} \mathcal{L}$.

Proof: Each $L \in \mathcal{C} \mathcal{L}$ is positive semidefinite and has $\lambda_{1}(L)=0$, with corresponding eigenvector $\overrightarrow{1}$. Thus $\lambda_{2}(L)$ may be expressed as

$$
\lambda_{2}(L)=\inf \left\{\vec{x}^{T} L \vec{x}:\|\vec{x}\|_{2}=1 \text { and } \overrightarrow{1}^{T} \vec{x}=0\right\} .
$$

For each $\vec{x} \in \mathbb{R}^{n}$ that satisfies $\|\vec{x}\|_{2}=1$ and $\overrightarrow{1}^{T} \vec{x}=0, \vec{x}^{T} L \vec{x}$ is a linear (and therefore also concave) function of $L$. The formula shows that $\lambda_{2}$ is the infimum of a family of concave functions in $L$, and is therefore also a concave function of $L$.

A generalization of Proposition 3 is also true and is proven in the same manner.

Proposition 4 ( [38]): The sum of the $k$ smallest eigenvalues of $L$,

$$
g(L)=\sum_{i=2}^{k} \lambda_{i}(L)
$$

is a concave function of $L$.

Recall Problem 1, but now consider matrices in $\mathcal{C L}$ rather than in $\mathcal{L}$. This relaxed problem is a reverse convex program.

Definition 1 ( [46]): The optimization problem

$$
\begin{gathered}
\min f(x) \\
\text { subject to } x \in D \backslash C
\end{gathered}
$$

is a reverse convex program when $f(x)$ is a convex function, $D$ is a closed convex set, $C$ is an open convex set, and $D$ and $C$ are given by explicit convex inequalities.

Proposition 5: Considering $L \in \mathcal{C} \mathcal{L}$, the optimization problem:

$$
\begin{gathered}
\max \lambda_{2}(L) \\
\text { subject to } \sum_{i=2}^{r+1} \lambda_{i}(L) \leq C_{r}
\end{gathered}
$$

is a reverse convex program.

Proof: Follows from Propositions 3 and 4.

One may note that without the wiring cost constraint, the natural relaxation would have been a convex program [31]. There are several standard techniques for solving reverse convex programs [46]-[48], which may be used for the relaxed wiring cost constrained algebraic connectivity problem. A suitable heuristic, such as rounding may be used to convert a solution for the relaxed problem into a solution for Problem 1.

\section{Discussion}

Problem 1 and Problem 2 formalize tradeoffs between functionality, as measured by convergence speed of distributed inference algorithms, and wiring cost, as measured under optimal placement. Using an enumerative strategy for the NP-complete problem, some optimal graphs for small $n$ were found. For some systems in biology [14], small $n$ results might be sufficient to provide insight, however many systems have large $n$. An approach based on reverse convex program relaxations may yield large networks that are close 
to optimal. Trying reverse convex optimization remains for future work, as does comparison of optimal networks to natural and random networks [43].

\section{ACKNOWLEDGMENT}

Discussions with J. M. F. Moura (Carnegie Mellon University), D. B. Chklovskii (Janelia Farm Research Campus, Howard Hughes Medical Institute), S. K. Mitter, S. Kar (Carnegie Mellon University), J. Xavier (Instituto Superior Técnico), A. Olshevsky, P. Parrilo, and V. K. Goyal are appreciated.

\section{REFERENCES}

[1] M. T. Gastner and M. E. J. Newman, "The spatial structure of networks," Eur. Phys. J. B, vol. 49, no. 2, pp. 247-252, Jan. 2006.

[2] —- "Shape and efficiency in spatial distribution networks," J. Stat. Mech., vol. 2006, no. 1, pp. 247-252, Jan. 2006.

[3] R. Olfati-Saber, J. A. Fax, and R. M. Murray, "Consensus and cooperation in networked multi-agent systems," Proc. IEEE, vol. 95, no. 1, pp. 215-233, Jan. 2007.

[4] S. Kar, S. Aldosari, and J. M. F. Moura, "Topology for distributed inference on graphs," IEEE Trans. Signal Process., vol. 56, no. 6, pp. 2609-2613, June 2008.

[5] J. Diaz, J. Petit, and M. Serna, "A survey of graph layout problems," ACM Comput. Surv., vol. 34, no. 3, pp. 313-356, Sept. 2002.

[6] K. M. Hall, "An $r$-dimensional quadratic placement algorithm," Manage. Sci., vol. 17, no. 3, pp. 219-229, Nov. 1970.

[7] D. B. Chklovskii, "Exact solution for the optimal neuronal layout problem," Neural Comput., vol. 16, no. 10, pp. 2067-2078, Oct. 2004.

[8] Y. Koren, "Drawing graphs by eigenvectors: Theory and practice," Comput. Math. Appl., vol. 49, no. 11-12, pp. 1867-1888, June 2005.

[9] A. J. Seary and W. D. Richards, "Spectral methods for analyzing and visualizing networks: An introduction," in Dynamic Social Network Modeling and Analysis: Workshop Summary and Papers, R. Breiger, K. Carley, and P. Pattison, Eds. Washington, DC: The National Academies Press, 2003, pp. 209-228.

[10] A. A. Rad, M. Jalili, and M. Hasler, "Efficient rewirings for enhancing synchronizability of dynamical networks," Chaos, vol. 18, no. 3, p. 037104, Sept. 2008.

[11] M. Barahona and L. M. Pecora, "Synchronization in small-world systems," Phys. Rev. Lett., vol. 89, no. 5, p. 054101, July 2002.

[12] L. Donetti, P. I. Hurtado, and M. A. Muñoz, "Entangled networks, synchronization, and optimal network topology," Phys. Rev. Lett., vol. 95 , no. 18 , p. 188701 , Oct. 2005.

[13] B. L. Chen, D. H. Hall, and D. B. Chklovskii, "Wiring optimization can relate neuronal structure and function," Proc. Natl. Acad. Sci. U.S.A., vol. 103, no. 12, pp. 4723-4728, Mar. 2006.

[14] V. A. Klyachko and C. F. Stevens, "Connectivity optimization and the positioning of cortical areas," Proc. Natl. Acad. Sci. U.S.A., vol. 100, no. 13, pp. 7937-7941, June 2003.

[15] M. Kaiser and C. C. Hilgetag, "Nonoptimal component placement, but short processing paths, due to long-distance projections in neural systems," PLoS Comput. Biol., vol. 2, no. 7, p. e95, July 2006.

[16] E. R. van Dam and W. H. Haemers, "Which graphs are determined by their spectrum?" Linear Algebra Appl., vol. 373, pp. 241-272, Nov. 2003.

[17] W. H. Haemers and E. Spence, "Enumeration of cospectral graphs," Eur. J. Comb., vol. 25, no. 2, pp. 199-211, Feb. 2004.

[18] F. M. Atay and T. Biyikoglu, "Graph operations and synchronization of complex networks," Phys. Rev. E, vol. 72, no. 1, p. 016217, July 2005.

[19] N. M. M. de Abreu, "Old and new results on algebraic connectivity of graphs," Linear Algebra Appl., vol. 423, no. 1, pp. 53-73, May 2007.

[20] C. Maas, "Transportation in graphs and the admittance spectrum," Discrete Appl. Math., vol. 16, no. 1, pp. 31-49, Jan. 1987.

[21] F. Goldberg, "Bounding the gap between extremal Laplacian eigenvalues of graphs," Linear Algebra Appl., vol. 416, no. 1, pp. 68-74, July 2006.

[22] V. Borkar and P. P. Varaiya, "Asymptotic agreement in distributed estimation," IEEE Trans. Autom. Control, vol. AC-27, no. 3, pp. 650655, June 1982.
[23] J. N. Tsitsiklis and M. Athans, "Convergence and asymptotic agreement in distributed decision problems," IEEE Trans. Autom. Control, vol. AC-29, no. 1, pp. 42-50, Jan. 1984.

[24] P. F. Swaszek and P. Willett, "Parley as an approach to distributed detection," IEEE Trans. Aerosp. Electron. Syst., vol. 31, no. 1, pp. 447-457, Jan. 1995.

[25] P. K. Varshney, Distributed Detection and Data Fusion. New York: Springer-Verlag, 1997.

[26] T. Berger, Z. Zhang, and H. Viswanathan, "The CEO problem," IEEE Trans. Inf. Theory, vol. 42, no. 3, pp. 887-902, May 1996.

[27] T. S. Han and S.-I. Amari, "Statistical inference under multiterminal data compression," IEEE Trans. Inf. Theory, vol. 44, no. 6, pp. 2300 2324, Oct. 1998

[28] V. Misra, V. K. Goyal, and L. R. Varshney, "High-resolution functional quantization," in Proc. Data Compression Conf. (DCC 2008), Mar. 2008, pp. 113-122.

[29] A. Olshevsky and J. N. Tsitsiklis, "Convergence rates in distributed consensus and averaging," in Proc. 45th IEEE Conf. Decision Control, Dec. 2006, pp. 3387-3392.

[30] L. Xiao and S. Boyd, "Fast linear iterations for distributed averaging," Syst. Control Lett., vol. 53, no. 1, pp. 65-78, Sept. 2004.

[31] A. Ghosh and S. Boyd, "Growing well-connected graphs," in Proc. 45th IEEE Conf. Decision Control, Dec. 2006, pp. 6605-6611.

[32] A. Hagberg and D. A. Schult, "Rewiring networks for synchronization," Chaos, vol. 18, no. 3, p. 037105, Sept. 2008.

[33] M. Jalili and A. A. Rad, "Comment on "rewiring networks for synchronization"," Chaos, vol. 19, no. 2, p. 028101, May 2009.

[34] S. Belhaiza, N. M. M. de Abreu, P. Hansen, and C. S. Oliveira, "Variable neighborhood search for extremal graphs. XI. bounds on algebraic connectivity," in Graph Theory and Combinatorial Optimization, D. Avis, A. Hertz, and O. Marcotte, Eds. New York: Springer, 2005, pp. $1-16$

[35] H. J. Landau, "Sampling, data transmission, and the Nyquist rate," Proc. IEEE, vol. 55, no. 10, pp. 1701-1706, Oct. 1967.

[36] L. R. Varshney, "Optimal information storage: Nonsequential sources and neural channels," S.M. thesis, Massachusetts Institute of Technology, Cambridge, MA, June 2006.

[37] R. Olfati-Saber and R. M. Murray, "Consensus problems in networks of agents with switching topology and time-delays," IEEE Trans. Autom. Control, vol. 49, no. 9, pp. 1520-1533, Sept. 2004.

[38] A. Ghosh and S. Boyd, "Upper bounds on algebraic connectivity via convex optimization," Linear Algebra Appl., vol. 418, no. 2-3, pp. 693-707, Oct. 2006.

[39] M. R. Garey and D. S. Johnson, Computers and Intractability: A Guide to the Theory of NP-Completeness. San Francisco: W. H. Freeman and Company, 1979.

[40] D. Mosk-Aoyama, "Maximum algebraic connectivity augmentation is NP-hard," Oper. Res. Lett., vol. 36, no. 6, pp. 677-679, Nov. 2008

[41] N. J. A. Sloane, "The on-line encyclopedia of integer sequences." [Online]. Available: http://www.research.att.com/ njas/sequences

[42] L. A. Goldberg, Efficient Algorithms for Listing Combinatorial Structures. Cambridge: Cambridge University Press, 1993.

[43] L. R. Varshney, "Unreliable and resource-constrained decoding," $\mathrm{Ph} . \mathrm{D}$. thesis, Massachusetts Institute of Technology, Cambridge, MA, June 2010.

[44] L. R. Varshney, B. L. Chen, E. Paniagua, D. H. Hall, and D. B. Chklovskii, "Structural properties of the Caenorhabditis elegans neuronal network," arXiv:0907.2373 [q-bio]., July 2009.

[45] R. Marculescu and P. Bogdan, "The chip is the network: Toward a science of network-on-chip design," Found. Trends Electron. Design Autom., vol. 2, no. 4, pp. 371-461, 2009.

[46] H. Konno, P. T. Thach, and H. Tuy, Optimization on Low Rank Nonconvex Structures. Dordrecht: Kluwer Academic Publishers, 1996.

[47] H. Tuy, Convex Analysis and Global Optimization. Dordrecht: Kluwer Academic Publishers, 1998.

[48] J.-B. Hiriart-Urruty, "Conditions for global optimality 2," J. Global Optim., vol. 13, no. 4, pp. 349-367, Dec. 1998. 